International Journal of Physical Research, $8(2)(2020) 45-49$
International Journal of Physical Research
SPC
Website: www.sciencepubco.com/index.php/IJPR
Research paper

\title{
Elastic constants and optical phonon frequencies of $B X(X=P$, As, and Sb) semiconductors: Semi-empirical prediction
}

\author{
Salah Daoud $^{1 *}$, Pawan Kumar Saini², Hamza Rekab-Djabri ${ }^{3,4}$ \\ ${ }^{1}$ Laboratory of Materials and Electronic Systems (LMSE), Faculty of Sciences and Technology, Mohamed Elbachir El Ibrahimi, Bordj \\ Bou Arreridj University, 34000 Bordj Bou Arreridj, Algeria \\ ${ }^{2}$ Department of Physics, Government College, Hansi, Haryana, 125033, India \\ ${ }^{3}$ Laboratory of Micro and Nanophysics (LaMiN), Oran ENP, BP 1523, El M'Naouer, 31000, Oran, Algeria \\ ${ }^{4}$ Faculty of Nature and Life Sciences and Earth Sciences, Akli Mohand-Oulhadj University, 10000, Bouira, Algeria \\ *Corresponding author_E-mail:salah_daoud07@yahoo.fr
}

\begin{abstract}
Based on some simple empirical formulas established by Adachi in, Properties of group-IV, III-V and II-VI semiconductors, John Wiley \& Sons, Chichester (2005), and the experimental lattice constants reported in the literature; the present work aims to predict the elastic constants and some other significant properties of cubic zinc-blende boron compounds (BP, BAs and BSb). The obtained values of $C_{12}$ and $C_{44}$ are in general good agreement with other data of the literature, while $C_{11}$ and $B$ are slightly lower. The zone-center longitudinal optical (LO) and transverse optical (TO) phonon frequencies are also obtained. The LO and TO phonon frequencies of BP compound were found at 866.6 and $834.5 \mathrm{~cm}^{-1}$, respectively; these of BAs were found at 731.3 and $727.1 \mathrm{~cm}^{-1}$, respectively; while for the BSb narrow-gap semiconducting compound were found at around 598.3 and $586.2 \mathrm{~cm}^{-1}$, respectively. These two later values are in general slightly lower than the calculated values, and the observed Raman spectroscopy values reported in the literature.
\end{abstract}

Keywords:Boron Compounds; Lattice Parameter; Elastic Constants; Dynamical Properties; Thermal Properties.

\section{Introduction}

Among group III-V semiconducting materials, binary boron compounds, which have attracted great attention in recent years. These materials have many applications in electronic devices, optical devices that operate in the short wavelength range of the visible spectrum and in devices operating at high temperatures [1].The majority of these materials crystallize in zincblende and wurtzite structures, and they are characterized by different degrees of covalent, ionic, and metallic bonding [2].

Boron with chemical symbol (B) and antimony with chemical symbol (Sb) are relatively two abundant elements in nature [3]. At room temperature, boron antimonite $(\mathrm{BSb})$ material crystallizes in cubic zinc-blende phase; it is narrow-gap semiconducting material with an indirect gap of around $0.52 \mathrm{eV}$ [3]. This band gap is smaller compared to that that of silicon (i.e. smaller than $1.11 \mathrm{eV}$ ), which perhaps make this material as good candidate in the fabrication of the infrared detectors or the thermoelectric devices.

Boron and antimony have very different atomic masses; this characteristic specifies a wide gap between optical and acoustical phonon branches [3]. Yao et al. [4] have investigated the electronic and phononic properties of bulk boron-antimonide material using Ab initio calculations. They conclude that BSb can be a good material candidate with low-cost and easily processed, for hot carrier absorbers (HCA) in high-efficiency solar cell.

Bouamama and coworkers [5] have used the density-functional perturbation theory (DFPT) in the framework first principles approach to investigate the high-pressure effects on the lattice dynamics and dielectric properties of the BN, BP, BAs, BSb and BBi binary compounds. They found that the calculated LO and TO phonon frequencies increase in quadratic form with pressure for all boron compounds. They also calculated the band structure of boron compounds along the high-symmetry directions in the Brillouin zone and found that all these materials have an indirect band gap. Lindsay and coworkers [6] have studied the thermal conductivity, the Debye temperature, and some other properties of III-V boron semiconducting compounds using first principles approach. They found that BSb material has a thermal conductivity near $465 \mathrm{Wm}^{-1} \mathrm{~K}^{-1}$, and a Debye temperature at around $495 \mathrm{~K}$.

In our previous work [7-11], the elastic constants, the electronic and thermal properties, the piezoelectric coefficients, the sound velocities and several other physical properties of cubic zinc-blende BSb and BAs materials at equilibrium and under high pressure were studied using ab-initio calculations and some other semi-empirical approaches.

Recently, Bioud et al. [12] have investigated the temperature and pressure effect on the thermodynamic properties of BSb using the quasi-harmonic Debye model. They also predict several other parameters such as: the electronic polarizability, the microhardness, the structural phase transition under compression, the melting temperature, the plasmon energy, and the thermal conductivity at $300 \mathrm{~K}$. 
Very recently, Malica and Dal Corso [13] have investigated the room temperature phonon dispersions and the temperature dependence on the elastic constants and thermodynamic properties of BAs using an ab initio approach. They also investigated the effect of the temperature on the sound velocity of the longitudinal acoustic mode along the [111] direction.

In the present work, we predicted the elastic constants, thermal and thermodynamic properties, as well as the longitudinal and transverse optical phonon frequencies of BP, BAs and BSb semiconducting compounds using a semi-empirical approach.

\section{Theory, calculations, results, and discussion}

\subsection{Crystal structure of BP, BAs and BSb binary compounds}

The lattice spacing between the atoms of crystal is an important parameter; it affects several physical quantities, such as the elastic constants, the width of the gap in the semiconductors, the volumetric mass density, etc[8]. Most binary III-V semiconducting compounds can crystallize in either cubic zincblende (B3) or hexagonal wurtzite (B4) structures [14]. Some other materials (such as BN and AlN) can crystallize in both structures at ambient conditions. The cell geometry of B3 configuration was assigned as: $a=b=c$ (lattice constants), and $\alpha=\beta=\gamma=90^{\circ}$ (lattice angles), while that of B4 phase was assigned as: $\mathrm{a}=\mathrm{b} \neq \mathrm{c}$ (lattice constants), $\mathrm{u}$ (the internal parameter), $\alpha=\beta$ $=90^{\circ}$, and $\gamma=120^{\circ}$ (lattice angles). In both structures, the material is constituted from two different atoms per basis. At ambient conditions, the boron based materials (BP, BAs and BSb compounds) crystallize in cubic zincblende structure [3, 14], with the coordinates of the two basis atoms are $(0,0,0)$ and $(1 / 4,1 / 4,1 / 4)$, respectively. For our materials of interest, the experimental values of the lattice parameter are: $(4.5383 \AA)$ [14] for BP, $(4.777 \AA)$ [14] for BAs, and ( 5.30 $)$ [3] for BSb, respectively.

\section{2. Elastic constants and their related properties}

The present section focuses on investigating the elastic constants $C_{\mathrm{ij}}$ and their related parameters such as the Zener anisotropy parameter $\mathrm{Z}$, the Knoop microhardness $\mathrm{H}$, the elastic wave speed $\mathrm{v}$, the Debye temperature $\theta_{\mathrm{D}}$ and the melting point $\mathrm{T}_{\mathrm{m}} \mathrm{O}$ BP, BAs and BSb compounds. For certain cubic group-IV, III-V and II-VI semiconductor, the elastic constants $\mathrm{C}_{\mathrm{ij}}$ and the lattice parameter a are related by the following expressions [14]:

$\ln \mathrm{C}_{11}=-4.59 \ln \mathrm{a}+10.33 ; \ln \mathrm{C}_{12}=-2.54 \ln \mathrm{a}+6.07 ; \ln \mathrm{C}_{44}=-5.20 \ln \mathrm{a}+10.59$

Where a is expressed in $\AA$, and the elastic stiffness constant $\mathrm{C}_{\mathrm{ij}}$ are expressed in $10^{10} \mathrm{~Pa}$, respectively.

The proposed relations of Eq. (1) have been applied to evaluate the elastic stiffness constants $\mathrm{C}_{\mathrm{ij}}$ of $\mathrm{BP}$, BAs and BSb compounds.

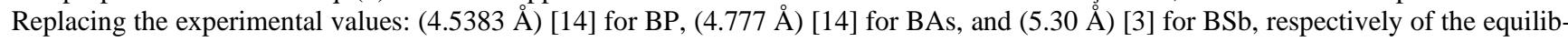
rium lattice parameters a in the relations of Eq. (1), the obtained values of elastic stiffness constants $\mathrm{C}_{11}, \mathrm{C}_{12}$, and $\mathrm{C}_{44}$ are summarized in Table 1, along the results obtained in Refs. [9], [14], [15] and [16]. In general, our values of the elastic constants $\mathrm{C}_{\mathrm{ij}}$ of $\mathrm{BP}$ and BAs are in agreement with other data of the literature. For BSb compound, except the value of $\mathrm{C}_{12}(62.59 \mathrm{GPa})$, the values of $\mathrm{C}_{11}(145.16)$ and $\mathrm{C}_{44}$ (68.07 GPa) are slightly lower than the theoretical ones 203.90 and $90.36 \mathrm{GPa}$, respectively as reported in Ref. [9].

The compressibility $\beta$ of material is defined as the reciprocal of the bulk modulus B. A substance that is difficult to compress has a small compressibility, but a large bulk modulus [7]. In cubic crystals, the bulk modulus B is expressed as a function of the elastic constants $\mathrm{C}_{11}$ and $\mathrm{C}_{12}$ as follow [13, 16]: $\mathrm{B}=\left(\mathrm{C}_{11}+2 \mathrm{C}_{12}\right) / 3$. Ourcalculated values of $\mathrm{B}$ for $\mathrm{BP}, \mathrm{BAs}$ and $\mathrm{BSb}$ are around 160.51, 132.28, and 90.11 GPa, respectively. They are also summarized in Table 1, along the results obtained in Refs. [9], [12], [14], [15] and [16]. Like the elastic constants $\mathrm{C}_{\mathrm{ij}}$, our values of the bulk modulus of BP and BAs are also in general in agreement with other data of the literature. For example the deviation between our value $(132.28 \mathrm{GPa})$ and the theoretical one $(129 \mathrm{GPa})$ reported by Tian et al. [16] is only around $2.54 \%$. Although the first $(160.51 \mathrm{GPa})$ and the second $(132.28 \mathrm{GPa})$ values are in general well agreement with other data of the literature, it is very clear that the later value $(90.11 \mathrm{GPa})$ is slightly lower than the theoretical values 106.8 and $108 \mathrm{GPa}$ reported in Ref. [9]. To the best of our knowledge there is no experimental data on the elastic constants $\mathrm{C}_{\mathrm{ij}}$ and the bulk modulus B for BSb compound.

The Cauchy's pressure $\mathrm{Cp}$ is quantity which used to study the ductility and brittleness of solids. This quantity is defined as the difference between $\mathrm{C}_{12}$ and $\mathrm{C}_{44}\left(\mathrm{Cp}=\mathrm{C}_{12}-\mathrm{C}_{44}\right)$. If $\mathrm{Cp}$ is positive (i.e. $\left.\mathrm{Cp}>0\right)$, the material is expected to be ductile in nature, and in another case, i.e. if $\mathrm{Cp}<0$, the material is expected to be brittle [17]. For BP, BAs and BSb compounds, our calculated values of Cp are: $-59.70,-35.34$, and -5.48 , respectively. Our obtained values of $\mathrm{Cp}$ are negative, which show that all these compounds behave as brittle materials.

In cubic diamond and cubic sphalerite structures, the relative position of the cation and anion sub-lattices under volume-conserving strain distortions for the positions which are not fixed by symmetry, was described by the Kleinman parameter (called also the internal strain parameter) $\zeta[18]$. For crystals with cubic diamond and cubic zinc-blende structures; the internal strain parameter and the elastic constants are related by following expression [18]: $\zeta=\left(\mathrm{C}_{11}+8 \mathrm{C}_{12}\right) /\left(7 \mathrm{C}_{11}+2 \mathrm{C}_{12}\right)$.For BP, BAs and $\mathrm{BSb}$ compounds, our calculated values of $\zeta$ are around: $0.46,0.49$, and 0.57 , respectively. Our obtained values of $\zeta$ are slightly higher than the previously calculated results $0.368,0.39$, and 0.445 , respectively reported by Ustundag et al. [18].

We can obtain different anisotropy parameters using the elastic stiffness constants. For cubic crystals, the Zener anisotropy parameter Z is usually used. This latter is expressed as function of the elastic stiffness constants $C_{\mathrm{ij}}$ as follow [7], [19]:

$\mathrm{Z}=2 \mathrm{C}_{44} /\left(\mathrm{C}_{11}-\mathrm{C}_{12}\right)$

For isotropic media $Z=1$, while if $Z \neq 1$ the crystal possesses an elastic anisotropy [19]. In addition, if $Z<1$, the crystal is stiffest along $<100>$ cube axes, and when $Z>1$, it is stiffest along the $<111>$ body diagonals [7]. The Zener anisotropy parameter based on our present reported elastic constants obtained for $\mathrm{BP}, \mathrm{BAs}$ and $\mathrm{BSb}$, respectively, 1.50, 1.53, and 1.65; which indicate that all these materials are stiffest along the $\langle 111\rangle$ body diagonal (the direction of the nearest-neighbor bond).Our calculated values of the Zener anisotropy ratio for BP, BAs and BSb are lower than the results: 2.64 and 2.35 reported for ZB-AlN and RS-AIN, respectively [19], which indicates the lowest elastic anisotropy in BP, BAs and BSb compared to AlN in both zinc-blende and rock-salt phases.

The hardness is related to the crystal structure of the material as well as the concentration of defects (dislocations, etc). There are different model related the hardness with other elastic moduli. For cubic group-IV, III-V and II-VI materials, the Knoop microhardness H and the bulk modulus B are related by the following linear formula [14]: 
$\mathrm{H}=0.16 \mathrm{~B}-5.74$

Replacing our obtained values of Bin this equation; the obtained values of $\mathrm{H}$ for BP, BAs and BSb are: 19.94, 15.42 and 8.68 GPa, respectively. They are also summarized in Table 1, along the results obtained in Refs. [9], [12], [14] and [16]. We note that the use of the empirical method slightly underestimates the Knoop microhardness H of BSb compared to the available values, while the discrepancy becomes more important for BP and BAs.

Table 1: Elastic Constants $\mathrm{C}_{\mathrm{ij}}$ (in GPa), Bulk Modulus B (in GPa), and Knoop Microhardness H (in GPa) of BP, BAs and BSb Semiconducting Compounds, Compared to Other Data [9], [12], [14 - 16]

\begin{tabular}{|c|c|c|c|c|c|c|}
\hline & & $\mathrm{C}_{11}$ & $\mathrm{C}_{12}$ & $\mathrm{C}_{44}$ & $\mathrm{~B}$ & $\mathrm{H}$ \\
\hline \multirow{3}{*}{ BP } & This work & 295.88 & 92.82 & 152.52 & 160.51 & 19.94 \\
\hline & Ref. [14] & 315 & 100 & 160 & 170 & 32 \\
\hline & Ref. [15] & 356 & 83.5 & 194.6 & 174.6 & - \\
\hline \multirow{4}{*}{ BAs } & Ref. [9] & 289.56 & 77.25 & 129.62 & - & $23.82(17.94)$ \\
\hline & Ref. [14] & 279 & 120 & 113 & 173 & 19 \\
\hline & Ref. [16] & 263 & 62 & 143 & 129 & 22 \\
\hline & This work & 145.16 & 62.59 & 68.07 & 90.11 & 8.68 \\
\hline \multirow{2}{*}{$\mathrm{BSb}$} & Ref. [9] & 203.90 & 60.05 & 90.36 & $106.8(108)$ & $9.73(11.49)$ \\
\hline & Ref. [12] & - & - & - & 95.30 & 9.80 \\
\hline
\end{tabular}

The Debye temperature $\theta_{\mathrm{D}}$ is a useful parameter in solid-state problems, this because of its inherent relationship to lattice vibration [14].The calculated structural parameters and elastic constants of crystals are usually used to predict their Debye temperature $\theta_{\mathrm{D}}[20]$. At low temperature, $\theta_{\mathrm{D}}$ can be obtained from the sound velocity [20]. For binary crystals with cubic zinc-blende structure, $\theta_{\mathrm{D}}$ can be obtained from the average sound velocity $\mathrm{v}_{\mathrm{m}}$ (expressed in $\mathrm{km} / \mathrm{s}$ ), and the lattice constant a (expressed in $\AA$ ) as follow [21], [22]:

$\theta \mathrm{D} \approx(595.467) \mathrm{Vm} / \mathrm{a}$

Using the same formulas used in our previous works [21, 22], the average sound velocities $\mathrm{V}_{\mathrm{m}}$ of BP, BAs and BSb semiconducting compounds were found at around:7.279, 4.793 and $3.404 \mathrm{~km} / \mathrm{s}$, respectively. Replacing these values of $\mathrm{Vm}_{\mathrm{m}}$ in Eq (4); the obtained value of $\theta_{\mathrm{D}}$ are around:955, 597 and $382 \mathrm{~K}$, respectively. Our obtained values of $\theta_{\mathrm{D}}$ for BP and BAs compounds are slightly lower than the experimental $1025 \mathrm{~K}$ (measured at $\mathrm{T}=320 \mathrm{~K}$ ) for BP [14], and $681 \mathrm{~K}$ for BAs [16], respectively. The deviation between our calculated value $(955 \mathrm{~K})$ and the experimental one $(1025 \mathrm{~K})$ reported in Ref. [14] is around $6.83 \%$. Although the Debye temperature $\theta_{\mathrm{D}}$ of BSb has been extensively studied by the theoretical calculation [6], [10], and [12]; to the best of our knowledge there is no experimental data on this parameter for BSb compound. Our computed value of $\theta_{\mathrm{D}}$ of BSb is slightly lower than the theoretical one 456.07 $\mathrm{K}$ reported in Ref. [10], but excellent agreement with the theoretical data $384.9 \mathrm{~K}$ reported by Bioud et al. [12]. We have also calculated the Debye temperature $\theta_{\mathrm{D}}$ using a semi-empirical formula expressed as follow [23]:

$\theta_{D}=C_{B}\left(a G_{B} / M\right)^{1 / 2}, G_{B}=\left[C_{44}\left(C_{11}-C_{12}\right)\left(C_{11}-C_{12}+2 C_{44}\right)\right]^{1 / 3}$

where $\mathrm{a}$ is the lattice parameter, $\mathrm{C}_{\mathrm{B}}=3.89 \times 10^{11} \mathrm{n}^{-1 / 6} \mathrm{~h} / \mathrm{k}_{\mathrm{B}}$ is a model parameter, $\mathrm{h}$ is Planck's constant, $\mathrm{k}_{\mathrm{B}}$ is Boltzmann's constant, $\mathrm{n}$ is the number of atoms in the unit cell, and $\mathrm{M}$ is the atomic weight (arithmetical average of the masses of the species), respectively.

Using Eq. (5), the Debye temperature $\theta_{\mathrm{D}}$ of BP, BAs and BSb semiconducting compounds were found at around: 1025.9, 644.4 and 416.6 $\mathrm{K}$, respectively. Although our values (1025.9and 644.4 K) of BP and BAs are slightly higher than the values (955 and $597 \mathrm{~K}$ ) obtained from Eq. (4), they are in very good agreement with the experimental ones (1025 K for BP [14], and $681 \mathrm{~K}$ for BAs [16]). The deviation between our calculated value $(1025.9 \mathrm{~K})$ of $\theta_{\mathrm{D}}$ and the experimental one $(1025 \mathrm{~K})$ reported by Adachi[12] is less than $0.09 \%$, while that between our value $(644.4 \mathrm{~K})$ and the experimental one $(681 \mathrm{~K})$ reported by Tian and co-workers [16] is only around $5.4 \%$.For BSb material, our obtained value of $\theta_{\mathrm{D}}$ is slightly higher than that $(382 \mathrm{~K})$ obtained from Eq. (4) and slightly lower than the theoretical data $495 \mathrm{~K}$ reported by Lindsay and coworkers [6] and the value $(491.88 \mathrm{~K})$ reported in our previous work [10], so it is localized between these two theoretical data.

The melting point is one of the most essential thermophysical parameters of solid[14]. There are different expressions usually used to predict the melting point $\mathrm{T}_{\mathrm{m}}$. For materials with cubic structure, the melting point $\mathrm{T}_{\mathrm{m}}$ correlates with the elastic constants $\mathrm{C}_{\mathrm{ij}}$ as follow [24]: $\mathrm{T}_{\mathrm{m}}=560.4+7.805 \mathrm{C}_{11}-3.094 \mathrm{C}_{12}-1.086 \mathrm{C}_{44}$, where the elastic constants $\mathrm{C}_{\mathrm{ij}}$ are expressed in $\mathrm{GPa}$, and the melting point $\mathrm{T}_{\mathrm{mis}}$ expressed in $\mathrm{K}$. Replacing our values of $\mathrm{C}_{\mathrm{ij}}$ in the previous expression, the melting point $\mathrm{T}_{\mathrm{m}}$ of $\mathrm{BP}$, BAs and BSb compounds were estimated at around: $2416.94,2006.59$ and $1425.8 \mathrm{~K}$, respectively. Like bulk modulus B and the microhardness $\mathrm{H}$, our values of $\mathrm{T}_{\mathrm{m}}$ for BP, BAs and BSb compounds are also slightly lower than the experimental (> $3300 \mathrm{~K}$ for BP [14], $2300 \mathrm{~K}$ for BAs [14]) and other theoretical $(1758.05 \pm 300 \mathrm{~K}$ and $1500 \mathrm{~K}$ reported in Ref. [10], and by Bioud et al. [12], respectively for BSb) values. The deviation less than 5\% between our calculated value $(1425.8 \mathrm{~K})$ of $\mathrm{T}_{\mathrm{m}}$ and the theoretical one $(1500 \mathrm{~K})$ reported by Bioud and coworkers [12].

\subsection{Thermodynamic properties}

Appling an approach based on the correlation between thermodynamic properties and melting point $\mathrm{T}_{\mathrm{m}}$, Vassiliev et al. [25] have calculated several thermodynamic properties of certain III-V semiconducting materials. The dependence of the standard enthalpy of formation $\Delta_{f} H_{298}^{0}$ and the standard entropy $S_{298}^{0}$ on melting point $\mathrm{T}_{\mathrm{m}}$ can be approximately described by the following equations [25]:

$\Delta_{f} H_{298}^{0}=11.38-0.0343 \mathrm{~T}_{\mathrm{m}}$

$S_{298}^{0}=167.664-18.68 \ln \left(\mathrm{T}_{\mathrm{m}}\right)$ 
Where the enthalpy of formation $\Delta_{f} H_{298}^{0}$ is expressed in $\mathrm{kJ} / \mathrm{mol}$-at, the standard entropy $S_{298}^{0}$ is expressed in $\mathrm{J} /(\mathrm{K}$ mol-at), while the melting point $\mathrm{T}_{\mathrm{m}}$ is expressed in $\mathrm{K}$.

The enthalpy of formation $\Delta_{f} H_{298}^{0}$ of BP, BAs and BSb semiconducting compounds were estimated at around:-71.52, -57.45 and -37.52 $\mathrm{kJ} / \mathrm{mol}$-at, respectively; while these of the standard entropy $S_{298}^{0}$ were found: $22.14,25.62$ and $32 \mathrm{~J} /(\mathrm{K}$ mol-at), respectively. Our value of $S_{298}^{0}$ for BAs semiconducting compound is slightly higher than the experimental values (from $15.76 \pm 0.6$ to $\left.20.5 \pm 2\right) \mathrm{J} /(\mathrm{K} \mathrm{mol}-\mathrm{at}) \mathrm{re}-$ ported by Vassiliev and coworkers [25].

\section{4. Longitudinal and transverse optical phonon frequencies}

Phonons play a major role in many physical properties of solids, such as thermal property, superconducting and electrical conductivity [26]. Under consideration of the scarce data on the dynamic properties of BP, BAs and BSb, the present work investigates the longitudinal and transverse optical phonon frequencies of BP, BAs and BSb materials. We have calculated the longitudinal optical (LO) and transverse optical (TO) phonon frequencies, following the method of Harrison [27] and Wolverson and coworkers [28]. The calculation of LO phonon frequency at the zone-center has been geared up using the following expression [23]:

$\omega_{L O}^{2}=\left(8 / 3 \mu d^{2}\right)\left(C_{o}+8 C_{1}\right)$

Where $\mu$ is the reduced mass of an $\mathrm{BX}(\mathrm{X}=\mathrm{P}, \mathrm{As}$, and $\mathrm{Sb})$ formula unit cell $\left(\mu=\mathrm{M}_{1} \cdot \mathrm{M}_{2} /\left(\mathrm{M}_{1}+\mathrm{M}_{2}\right)\right), \mathrm{d}$ is the interatomic separation, $\mathrm{C}_{0}$ is the bond stretching force constant and $\mathrm{C}_{1}$ is the bond bending force constant. The force constants can be expressed:

$C_{0}=\left(3 a^{3} / 16\right)\left(C_{11}+2 C_{12}\right)$, and $C_{1}=\left(a^{3} / 32\right)\left(C_{11}-C_{12}\right)$

The zone-center LO phonon frequency of BP, BAs, and BSb using this procedure has been obtained as: $866.6,731.3$ and $598.3 \mathrm{~cm}^{-1}$, respectively. They are also summarized in Table 2, along the results obtained in Refs. [3], [5], [14] and [29]. Our value (598.3 $\left.\mathrm{cm}^{-1}\right)$ of the zone-center LO of BSb is slightly lower than the calculated values $621,623 \mathrm{~cm}^{-1}$, and the observed Raman spectroscopy value 623 $\mathrm{cm}^{-1}$ reported by Touat et al. [29], Bouamama and coworkers [5], and Das et al. [3], respectively. The zone-center TO phonon frequency can be calculated by the Lydanne - Sachs -Teller relation [30] (well-known LST relation), which is expressed as follow [31]:

$\omega_{T}^{2}=(\varepsilon(\infty) / \varepsilon(0)) \omega_{L}^{2}$

Where $\omega_{\mathrm{T}}$ is the transverse optical frequency, $\omega_{\mathrm{L}}$ is the longitudinal optical frequency (both at low wave vector), $\varepsilon(\infty)$ is the highfrequency limit of the dielectric constant and $\varepsilon(0)$ is the low-frequency (static) limit.

Replacing in Eq. (10) the values 10.2 and 11 of $\varepsilon(\infty)$ and $\varepsilon(0)$ reported by Adachi [14] for BP, the values 9.56 and 9.67 of $\varepsilon(\infty)$ and $\varepsilon(0)$ reported by Bouamama et al. [5], and Labidi et al. [32],respectively for BAs, and the values 10.72 and 11.17 of $\varepsilon(\infty)$ and $\varepsilon(0)$ reported by Bouamama et al. [5], and Labidi et al. [32],respectively for BSb; the zone-center TO phonon frequency of BP, BAs and BSb semiconducting compounds have been obtained as : 834.5, 727.1 and $586.2 \mathrm{~cm}^{-1}$, respectively. These values are also summarized in Table 2, along the results obtained in Refs. [3], [5], [14] and [29].

The value $586.2 \mathrm{~cm}^{-1}$ is slightly lower than the calculated values 611 and605 cm $\mathrm{cm}^{-1}$, and the observed Raman value $605 \mathrm{~cm}^{-1}$ reported by Touat et al. [29], Bouamama et al. [5], and Das et al. [3], respectively. The optical phonon frequency difference $\Delta \omega_{\mathrm{op}}=\omega_{\mathrm{LO}}-\omega_{\mathrm{TO}}$ of BP is $32.1 \mathrm{~cm}^{-1}$, that of BAs is $4.2 \mathrm{~cm}^{-1}$, while in case of BSb (narrow-gap semiconducting compound) has been obtained at around12.1 $\mathrm{cm}^{-1}$. They are also summarized in Table 2, along the results obtained in Refs. [3], [5], [14] and [29].

Table 2: Longitudinal Optical (LO) and Transverse Optical (TO) Phonon Frequencies ( $\mathrm{In}^{-1}$ ) of BP, BAs and BSb Compounds, Compared to Other Data [3], [5], [14], [29]

\begin{tabular}{lllll}
\hline & & & $\omega_{\text {TO }}$ & $\Delta \omega_{\text {op }}$ \\
\hline \multirow{3}{*}{ BP } & This work & $\omega_{\text {LO }}$ & 834.5 & 3.1 \\
& Ref. [14] & 866.6 & 798.9 & 4.2 \\
BAs & This work & 828.9 & 727.1 & 4 \\
& Ref. [5] & 731.3 & 701 & 12.1 \\
& This work & 705 & 586.2 & 18 \\
BSb & Ref. [3] Exp & 598.3 & 605 & 18 \\
& Ref. [5] & 623 & 605 & 10 \\
\hline
\end{tabular}

From data reported in Table 2, we can notice that our values of $\Delta \omega_{\mathrm{op}}$ are in good agreement with other results reported in Refs. [3], [5], [14] and [29]. For example, the deviation between our value $\left(32.1 \mathrm{~cm}^{-1}\right)$ and the value $\left(30 \mathrm{~cm}^{-1}\right)$ reported by Adachi [14] is 7\%, while the deviation between our value $\left(4.2 \mathrm{~cm}^{-1}\right)$ and the value $\left(4 \mathrm{~cm}^{-1}\right)$ reported by Bouamama et al. [5] is around $5 \%$ only.

\section{Conclusion}

Based on some simple semi-empirical and empirical formulas, and some experimental and theoretical data reported in literature, we tried to predict the elastic constants and related properties of cubic zinc-blende BP, BAs and BSb materials. Except the values of $\mathrm{C}_{12}$ and $\mathrm{C}_{44}$, these of $\mathrm{C}_{11}$ and $\mathrm{B}$ are slightly lower than the experimental and other theoretical values reported in the literature.

The Kleinman parameter was determined. In general, our obtained values are slightly higher than other calculated results of the literature. The analyses of the Zener anisotropy parameter indicates that BP, BAs and BSb are stiffest along the <111> body diagonals, while the Cauchy's pressure shows that all these compounds behave as brittle materials.

The Debye temperature was also calculated using two different formulas; generally the data obtained in this work are in agreement with other experimental and theoretical values of the literature. The standard enthalpy of formation, the entropy, the Knoop microhardness, the 
melting point, and the zone-center longitudinal optical (LO) and transverse optical (TO) phonon frequencies were also obtained. Except the standard entropy, our obtained values of other parameters are slightly lower than the experimental values and other calculated data of the literature.

\section{References}

[1] A. Rastogi, P. Rajpoot, and U. P. Verma, "Properties of group III-V semiconductor: BAs", Bulletin of Materials Science, Vol. 42, No. 3, (2019), 112 (11pp). https://doi.org/10.1007/s12034-019-1758-8.

[2] A. S. Verma, "Extant ionic charge theory for bond orbital model based on the tight-binding method: A semi-empirical model applied to wide-band gap II-VI and III-V semiconductors", Materials Science in Semiconductor Processing, Vol. 29, No. 1, (2015), pp. 2-15. https://doi.org/10.1016/j.mssp.2014.05.033.

[3] S. N. Das, R. Bhunia, S. Hussain, R. Bhar, B.R. Chakraborty, and A.K. Pal, " Synthesis and characterization of boron antimonide films by pulsed laser deposition technique", Applied Surface Science, Vol. 353, No. 6,(2015), pp. 439- 448. https://doi.org/10.1016/j.apsusc.2015.06.157.

[4] Y. Yao, D. König, and M. Green, "Investigation of boron antimonide as hot carrier absorber material ", Solar Energy Materials and Solar Cells, Vol. 111, (2014), pp. 123-126. https://doi.org/10.1016/j.solmat.2012.12.029.

[5] K. Bouamama, P. Djemia, N. Lebgaa and K. Kassali, "Ab initio calculation of the lattice dynamics of the Boron group-V compounds under high pressure", High Pressure Research, Vol. 27, No. 2 (2007), pp. 269 -277. https://doi.org/10.1080/08957950701265359.

[6] L. Lindsay, D. A. Broido, and T. L. Reinecke, "First-principles determination of ultrahigh thermal conductivity of boron arsenide: A Competitor for Diamond? “Physical Review Letters, Vol. 111, No. 2, (2013), 025901 (5pp). https://doi.org/10.1103/PhysRevLett.111.025901.

[7] S. Daoud, N. Bioud, N. Lebga, L. Belagraa and R. Mezouar, " Pressure effect on structural, elastic and electronic properties of (B3) BSb compound ", Indian Journal of Physics, Vol. 87, No.4, (2013), pp. 355-362.https://doi.org/10.1007/s12648-012-0231-y.

[8] S. Daoud, "Structural and piezoelectric properties of BSb under high pressure: A DFT study ", Journal of Nano-and Electronic Physics: Vol. 11, No.5, (2019), pp. 05004 (4pp). https://doi.org/10.21272/jnep.11(5).05004.

[9] S. Daoud, "Empirical study of elastic properties of BX (X = As, Sb) materials ", International Journal of Scientific World, Vol.3, No.1, (2015), pp. 37-42.https://doi.org/10.14419/ijsw.v3i1.4022.

[10] S. Daoud, "Sound velocities and thermal properties of BX (X=As, Sb) compounds", International Journal of Scientific World, Vol.3, No.1, (2015), pp. 43-48.https://doi.org/10.14419/ijsw.v3i1.4039.

[11] S. Daoud and A. Latreche, "Optical properties and electronic polarizability of boron-antimonide semiconductor ", International Journal of Physical Research: Vol. 5, No.2, (2017), pp. 43-45. https://doi.org/10.14419/ijpr.v5i2.7910.

[12] N. Bioud, X-W. Sun, S. Daoud, T. Song, and Z-J. Liu, "Structural stability and thermodynamic properties of BSb under high pressure and temperature", Materials Research Express, Vol. 5, No. 8, (2018) 085904 (12 pp). https://doi.org/10.1088/2053-1591/aad3a5.

[13] C. Malica, and A. Dal Corso, "Temperature dependent elastic constants and thermodynamic properties of BAs: An ab initio investigation", Journal of Applied Physics, Vol. 127, No.24, (2020), pp. 245103 (6pp).https://doi.org/10.1063/5.0011111.

[14] S. Adachi, "Properties of group-IV, III-V and II-VI semiconductors", John Wiley \& Sons Ltd, England, (2005). ISBN 0-470-09032-4. https://doi.org/10.1002/0470090340.

[15] S. Daoud, N. Bioud, L. Belagraa, and N. Lebga, "Elastic, optoelectronic and thermal properties of boron phosphide ", Journal of Nano-and Electronic Physics: Vol. 5, No.4, (2013), pp. 04061 (6pp). https://jnep.sumdu.edu.ua/download/numbers/2013/4/articles/jnep_2013_V5_04061.pdf.

[16] F. Tian, K. Luo, C. Xie, B. Liu, X. Liang, L. Wang, G. A. Gamage, H. Sun, H. Ziyaee, J. Sun, Z. Zhao, B. Xu, G. Gao, X.-F. Zhou, and Z. Ren, "Mechanical properties of boron arsenide single crystal ", Applied Physics Letters, Vol. 114, No.13, (2019), pp. 131903 (5 pp). https://doi.org/10.1063/1.5093289.

[17] S. Daoud, "Mechanical properties of BBi compound under pressure", International Journal of Scientific World, Vol.3, No.1, (2015), pp. 69-75. https://doi.org/10.14419/ijsw.v3i1.4218.

[18] M. Ustundag, M. Aslan, and Battal G. Yalcin, "The first-principles study on physical properties and phase stability of Boron-V (BN, $\mathrm{BP}$, BAs, BSb and BBi) compounds", Computational Materials Science, Vol.81, No.1, (2014), pp. 471-477. https://doi.org/10.1016/j.commatsci.2013.08.056.

[19] P. Verma, and P. S. Bisht, "Ab-initio study of AlN in zinc-blende and rock-salt phases ", Solid State Sciences, Vol.12, No.5, (2010), pp. 665-669. https://doi.org/10.1016/j.solidstatesciences.2008.12.002.

[20] S. Daoud, K. Loucif, N. Bioud, and N. Lebga, "First-principles study of structural, elastic and mechanical properties of zinc-blende boron nitride (B3-BN) ", Acta Physica Polonica A, Vol. 122, No. 1, (2012), pp.109-115. https://doi.org/10.12693/APhysPolA.122.109.

[21] S. Daoud, "Mechanical and piezoelectric properties, sound velocity and Debye temperature of thallium-phosphide under pressure", International Journal of Advanced Research in Physical Science, Vol. 1, No. 6, (2014), pp. 1-11. www.arcjournals.org/pdfs/ijarps/v1i6/1.pdf.

[22] S. Daoud, "Sound velocities and thermal properties of BeX (X=S, Se and Te) alkaline-earth chalcogenides", International Journal of Scientific World, Vol.5, No.1, (2017), pp. 9-12.https://doi.org/10.14419/ijsw.v5i1.6929.

[23] P. K. Jha, and M. Talati, "Mechanical, elastic and anharmonic properties of zinc blende MgS compound ", Physica Status Solidi B, Vol. 239, No. 2, (2003), pp. 291-296. https://doi.org/10.1002/pssb.200301829.

[24] T. Özer, "Study of first principles on anisotropy and elastic constants of $\mathrm{YAl}_{3}$ compound", Canadian Journal of Physics, Vol. 98, No. 4, (2020), pp. 357-363.https://doi.org/10.1139/cjp-2018-0448.

[25] V. P. Vassiliev, B. Legendre,and V. P. Zlomanov, "The critical analysis and mutual coherence of the thermodynamic data of the $\mathrm{A}^{\mathrm{III}} \mathrm{B}^{\mathrm{V}}$ phases", Intermetallics, Vol. 19, No. 12, (2011), pp. 1891-1901. https://doi.org/10.1016/j.intermet.2011.07.023.

[26] J. Tan, G. Ji, X. Chen, L. Zhang, and Y. Wen, "The high-pressure phase transitions and vibrational properties of zinc-blende XTe (X = Zn, Cd, Hg) Performance of local-density-approximation density functional theory", Computational Materials Science Vol. 48, No. 4, (2010), pp. 796801.https://doi.org/10.1016/j.commatsci.2010.03.037.

[27] W. A. Harrison, "Electronic structure and the properties of solids", W.H. Freeman, San Francisco, (1980). (reprinted by Dover, New York, 1988).

[28] D. Wolverson, D. M. Bird, C. Bradford, K. A. Prior, and B. C. Cavenett, " Lattice dynamics and elastic properties of zinc-blende MgS", Physical Review B, Vol. 64, No. 11, (2001) 113203 (xx pp). https://doi.org/10.1103/PhysRevB.64.113203.

[29] D. Touat, M. Ferhat and A. Zaoui, "Dynamical behaviour in the boron III-V group: a first-principles study", Journal of Physics: Condensed Matter, Vol. 18, No. 15, (2006), pp. 3647-3654. https://doi.org/10.1088/0953-8984/18/15/011.

[30] R. M. Lyddane, R. G. Sachs, and E. Teller, "On the polar vibrations of alkali halides", Physical Review, Vol. 59, No. 8, (1941), pp. 673676. https://doi.org/10.1103/PhysRev.59.673.

[31] S. Daoud, A. Bencheikh, and L. Belagraa, "Quasi-linear correlation between high-frequency and static dielectric constants in II-VI and III-V semiconductors ", International Journal of Physical Research, Vol. 5, No.1, (2017), pp. 4-6. https://doi.org/10.14419/ijpr.v5i1.6961.

[32] S. Labidi, H. Meradji, S. Ghemid, S. Meçabih, and B. Abbar, "Pressure dependence of electronic and optical properties of zincblende BP, BAs and BSb compounds", Journal of Optoelectronics and Advanced Materials, Vol. 11, No. 7, (2009), pp. 994 - 1001. http://joam.inoe.ro/index.php?option=magazine \&op=view\&idu=19 89\&catid=40. 